\title{
Assigning responsibility and credit
}

\author{
New policies refine the responsibilities of authors and require author contribution statements in Nature Chemical \\ Biology research papers.
}

C ontemporary scientific research is, at its core, a collaborative venture. Whether it involves colleagues from a single laboratory or a multidisciplinary team from several institutions, science advances through the combined efforts of individual researchers who contribute ideas, experiments and data analysis to ongoing projects. Published articles in peer-reviewed scientific journals communicate these discoveries to the broader community, but are also essential for the career development of individual scientists. Accordingly, it is critically important that all scientists assume responsibility for the integrity of their published work and ensure that they are properly acknowledged for their contributions. To facilitate these aims, Nature Chemical Biology and the other Nature journals have recently updated our authorship policies to clarify the responsibilities of authors and to ensure that the individual contributions of authors are accurately reflected in the published record (Nature 458, 1078, 2009).

When a manuscript is submitted to Nature Chemical Biology, one author assumes the role of corresponding author. This individual, typically a principal investigator of the study, serves as the central point of contact for the manuscript and manages communication with the journal while the paper is under consideration. Corresponding authors also take responsibility for coordinating communication among the paper's authors and for certifying that all authors have agreed to the contents of the manuscript prior to submission. Our updated authorship policy now clarifies the corresponding author's role in managing the manuscript's author list: prior to submission, the corresponding author "ensures that all authors are included in the author list, its order has been agreed by all authors, and that all authors are aware that the paper was submitted" (http://www. nature.com/authors/editorial_policies/authorship.html).

Why are these changes needed? Because publication records remain an important metric for assessing the research productivity of individual scientists, it is essential that author lists are accurate and are determined in an objective and open manner. Most authorship disputes result from lack of clarity on two main points: (i) defining whether an individual's scientific contributions warrant authorship and (ii) determining the order of the author list. Obtaining agreement on these points is made more challenging by differences in authorship models among disciplines and individual laboratories, and by the fact that collaborative papers in interdisciplinary areas often include data from multiple research groups.

Nature Chemical Biology does not legislate how individual investigators or collaborations should define authorship or determine author order, nor do we resolve authorship disputes. However, we do require written approval from all authors for any changes made to the composition or order of the author list after submission. To help authors comply with this policy, we routinely examine author lists of revised manuscripts and request that corresponding authors clarify any changes. More generally, we urge all principal investigators to establish and communicate clear authorship guidelines in their research groups and institute transparent mechanisms for resolving conflicts. We strongly suggest that collaborative research teams explicitly discuss authorship at the outset of multigroup projects. Even with good planning, all parties must realize that new results may alter the scope of a scientific study, and the peer review process may shift the focus of manuscripts or require additional experiments to address technical concerns. Evolving projects and manuscripts may alter working author lists, so principal investigators need to be attentive and renew authorship discussions as key project milestones are reached. Clear expectations and open dialog will not prevent all authorship misunderstandings, but they should provide channels necessary for resolving them.

In interdisciplinary fields such as chemical biology, many manuscripts involve contributions of data from multiple research groups with diverse scientific expertise. A second change to our authorship policies addresses the responsibilities of senior members of these collaborating teams. Under the new policy, for collaborative papers, a senior researcher from each group assumes responsibility for the team's contributions to the project. In doing so the senior author certifies that the data in the paper are representative of the original data, which have been appropriately archived, and that the team complies with our policies on sharing of data and materials. These new guidelines do not lessen the importance of oversight by the corresponding author, but they ensure that each senior scientist of a multigroup collaboration accepts responsibility for the primary data generated by his or her group.

Our final authorship policy change concerns 'author contribution' statements, which are short statements at the end of research papers that provide authors with a forum to highlight the contributions that each author made to the article. We believe that these statements offer an effective way to assign credit for individual achievements leading to a scientific advance. Beginning in 2006, authors of Nature Chemical Biology papers were encouraged to include an author contribution section, and most authors have voluntarily done so. Under our new guidelines (Nature $458,1078,2009)$, all Nature journals now require that authors of original research papers include an author contribution statement. Authors are free to format their declarations as they choose (for examples, see http:// blogs.nature.com/nautilus/2007/11/post_12.html), but we require that all authors are included in the statement. We feel that these required statements enhance the transparency of scientific papers, making it easier to assess each scientist's role in the discovery. Further delineating individual contributions in multigroup collaborations may go a long way toward resolving authorship issues by providing an additional dimension to the linear author lists of current papers.

Taken together, we feel that these policies, which more clearly spell out the roles of lead authors and formalize the author contribution process, offer benefits to all who contribute to and read the journal. We hope that you agree, and we welcome your feedback on these changes. 